\title{
The Kinetic Umbrella: Designing a transformable semi-elastic grid structure
}

\author{
J. Schikore*, E. Schling ${ }^{\dagger}$ \\ *Technical University of Munich \\ Department of Architecture - Chair of Structural Design, Arcisstr. 21, 80333 Munich \\ jonas.schikore@tum.de \\ † The University of Hong Kong, Department of Architecture, Pokfulam Road, Hong Kong
}

\begin{abstract}
The deformability of grid structures can be controlled using geometric and mechanical constraints to realize elastic mechanisms. The morphology of elastic grid structures has been related to differential geometry more than a century ago [1]. Today, numerical methods allow precise physical simulations of such compliant mechanisms including mechanical parameters. However, architectural applications are limited to static strained gridshells using elastic timber or steel profiles [2]. The design of transformable structures of this kind requires associated strategies [3].

In scope of this paper are quadrilateral grid structures with the ability to transform by semielastic mechanisms. We investigate strategies and workflows to design, simulate, analyse, and build such structures.
\end{abstract}

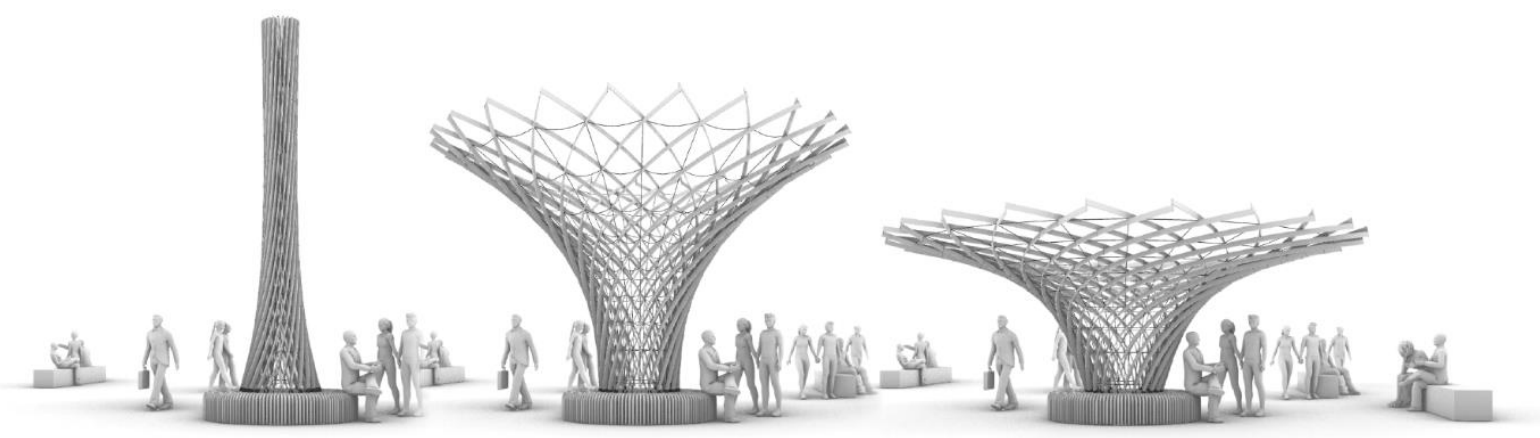

Figure 1. Stages of a transformable semi-elastic grid structure

We evaluate decisive parameters to control the transformation process of semi-compliant grid structures using actuation- and locking mechanisms, utilizing the grid members strain energy levels. We have a closer look on the material and section properties and their limiting parameters in context of large grid deformation. The practical range of profiles for such applications and the impact on the deformation performance is determined. We give insight into the planning workflow of the Kinetic Umbrella, a transformable research pavilion, from the first concept to detailed design.

\section{REFERENCES}

[1] S. Finsterwalder: "Mechanische Beziehungen bei der Flächen-deformation", Report by german mathmatician union, vol. 6, pp. 1-59, 1899.

[2] E. Schling, M. Kilian, J. Schikore, H. Pottmann: "Design and construction of curved support structures with repetitive parameters" in Conference Proceedings Advances in Architectural Geometry 2018 (AAG), Gothenburg, Sweden, September 2018

[3] J. Schikore, E. Schling, T. Oberbichler, A. M. Bauer: "Kinetics and Design of Semi-Compliant Grid Mechanisms" in Conference Proceedings Advances in Architectural Geometry 2020 (AAG), Paris, France, April 2021 